University of New Mexico

UNM Digital Repository

Mathematics and Statistics Faculty and Staff

Publications

Academic Department Resources

$9-2013$

\title{
Examples where the Conjunctive and Dempster's Rules are Insensitive
}

Florentin Smarandache

University of New Mexico, smarand@unm.edu

Jean Dezert

jean.dezert@onera.fr

Valeri Kroumov

Follow this and additional works at: https://digitalrepository.unm.edu/math_fsp

Part of the Computational Engineering Commons, Digital Communications and Networking

Commons, Logic and Foundations Commons, Other Computer Engineering Commons, Other Engineering

Commons, and the Other Mathematics Commons

\section{Recommended Citation}

Smarandache, Florentin; Jean Dezert; and Valeri Kroumov. "Examples where the Conjunctive and Dempster's Rules are Insensitive." Proc. of 2013 International Conference on Advanced Mechatronic Systems, Luoyang, China, Sept. 25-27, 2013 (2013): 211-213. https://digitalrepository.unm.edu/math_fsp/ 472

This Article is brought to you for free and open access by the Academic Department Resources at UNM Digital Repository. It has been accepted for inclusion in Mathematics and Statistics Faculty and Staff Publications by an authorized administrator of UNM Digital Repository. For more information, please contact amywinter@unm.edu, Isloane@salud.unm.edu, sarahrk@unm.edu. 


\title{
Examples where the Conjunctive and Dempster's Rules are Insensitive
}

\author{
Florentin Smarandache \\ Jean Dezert \\ Valeri Kroumov
}

\begin{abstract}
Originally published as Smarandache F., Kroumov V., Dezert J., Examples where the conjunctive and Dempster's rules are insensitive, Proc. of 2013 International Conference on Advanced Mechatronic Systems, Luoyang, China, Sept. 25-27, 2013, and reprinted with permission.
\end{abstract}

\begin{abstract}
In this paper we present several counter-examples to the Conjunctive rule and to Dempster rule of combinations in information fusion.
\end{abstract}

Keywords- conjunctive rule, Dempster rule, DSmT, counterexamples to Conjunctive rule, counter-examples to Dempster rule, information fusion

\section{INTRODUCTION}

In Counter-Examples to Dempster's Rule of Combination \{Ch. 5 of Advances and Applications to DSmT on Information Fusion, Vol. I, pp. 105-121, 2004\} [1], J. Dezert, F. Smarandache, and M. Khoshnevisan have presented several classes of fusion problems which could not be directly approached by the classical mathematical theory of evidence, also known as Dempster-Shafer Theory (DST), either because Shafer's model for the frame of discernment was impossible to obtain, or just because Dempster's rule of combination failed to provide coherent results (or no result at all). We have showed and discussed the potentiality of the DSmT combined with its classical (or hybrid) rule of combination to attack these infinite classes of fusion problems.

We have given general and concrete counter-examples for Bayesian and non-Bayesian cases.

In this article we construct new classes where both the conjunctive and Dempster's rule are insensitive.

\section{DEZERT-TCHAMOVA COUNTER-EXAMPLE}

In [2], J. Dezert and A. Tchamova have introduced for the first time the following counter-example with some generalizations. This first type of example has then been discussed in details in $[3,4]$ to question the validity of foundations of Dempster-Shafer Theory (DST). In the next sections of this short paper, we provide more counter-examples extending this idea. Let the frame of discernment $\Theta=\{\mathrm{A}, \mathrm{B}$, $C$, under Shafer's model (i.e. all intersections are empty), and $m_{1}($.$) and m_{2}($.$) be two independent sources of information that$ give the below masses:

\begin{tabular}{|c|c|c|c|c|}
\hline Focal Elements & $A$ & $C$ & $A \cup B$ & \multicolumn{2}{|c|}{$A \cup B \cup C$} \\
\hline$m_{1}$ & $a$ & 0 & $1-a$ & 0 \\
\hline$m_{2}$ & 0 & $1-b_{1}-b_{2}$ & $b_{1}$ & $b_{2}$ \\
\hline
\end{tabular}

Table 1

where the parameters $a, b_{1}, b_{2} \in[0,1]$, and $b_{1}+b_{2} \leq 1$.

Applying the conjunctive rule, in order to combine $\mathrm{m}_{1} \oplus \mathrm{m}_{2}=\mathrm{m}_{12}$, one gets:

$$
\begin{aligned}
& m_{12}(A)=a\left(b_{1}+b_{2}\right) \\
& m_{12}(C)=0 \\
& m_{12}(A \cup B)=(1-a)\left(b_{1}+b_{2}\right) \\
& m_{12}(A \cup B \cup C)=0
\end{aligned}
$$

and the conflicting mass

$$
m_{12}(\phi)=1-b_{1}-b_{2}=K_{12} \text {. }
$$

After normalizing by diving by $1-K_{12}=b_{1}+b_{2}$ one gets Demspter's rule result $m_{D S}($.$) :$

$$
\begin{aligned}
& m_{D S}(A)=\frac{m_{12}(A)}{1 K_{12}}=\frac{a\left(b_{1}+b_{2}\right)}{b_{1}+b_{2}}=a=m_{1}(A) \\
& m_{D S}(A \cup B)=\frac{m_{12}(A \cup B)}{1 K_{12}}=\frac{(1-a)\left(b_{1}+b_{2}\right)}{b_{1}+b_{2}}=1-a=m_{1}(A \cup B)
\end{aligned}
$$

Counter-intuitively after combining two sources of information, $m_{l}($.$) and m_{2}($.$) , with Dempster's rule, the result$ does not depend at all on $m_{2}($.$) . Therefore Dempster's rule is$ insensitive to $m_{2}\left(\right.$.) no matter what the parameters $a, b_{1}, b_{2}$ are equal to. 


\section{FUSION SPACE}

In order to generalize this counter-example, let's start by defining the fusion space.

Let $\Theta$ be a frame of discernment formed by $n$ singletons Ai, defined as:

$$
\Theta=\left\{\phi_{1}, \phi_{2}, \ldots, \phi_{n}\right\}, n \geq 2,
$$

and its Super-Power Set (or fusion space):

$$
S^{\Theta}=(\Theta, \cup, \cap, 0
$$

which means the set $\Theta$ closed under union $\cup$, intersection $\cap$, and respectively complement $\mathrm{C}$.

\section{ANOTHER CLASS OF COUNTER-EXAMPLES TO DEMPSTER'S RULE}

Let $A_{1}, A_{2}, \ldots, A_{p} \in S^{\Theta} \backslash\left\{I_{t}, \phi\right\}$, for $p \geq 1$, such that $A i \cap A j=\phi$ for $i \neq j$, where $I_{t}$ is the total ignorance $\left(A_{1} \cup A_{2} \cup \ldots \cup A_{n}\right)$, and $\phi$ is the empty set.

Therefore each $A_{i}$, for $i \in\{1,2, \ldots, p\}$, can be either a singleton, or a partial ignorance (union of singletons), or an intersection of singletons, or any element from the Super-Power Set $S^{\Theta}$ (except the total ignorance or the empty set), i.e. a general element in the set theory that is formed by the operators $\cup, \cap, \mathrm{C}$.

Let's consider two sources $m_{l}($.$) and m_{2}($.$) defined on S^{\Theta}$ :

$\begin{array}{llllll} & A_{1} & A_{2} & \cdots & A_{p} & I_{t} \\ m_{1} & a_{1} & a_{2} & \cdots & a_{p} & 0 \\ m_{2} & b & b & \cdots & b & 1-p \cdot b\end{array}$

where of course all $a_{i} \in[0,1]$ and $a_{1}+a_{2}+\ldots+a_{p}=1$, also $b$ and $1-p \cdot b \in[0,1]$.

$m_{l}($.) can be Bayesian or non-Bayesian depending on the way we choose the focal elements $A_{1}, A_{2}, \ldots, A_{p}$.

We can make sure $m_{2}($.$) is not the uniform basic believe$ assignment by setting $b \neq 1-p \cdot b$.

Let's use the conjunctive rule for $m_{1}$ (.) and $m_{2}($.$) :$

$m_{12}\left(A_{i}\right)=m_{1}\left(A_{i}\right) m_{2}\left(A_{i}\right)+\left[m_{1}\left(A_{i}\right) m_{2}\left(I_{t}\right)+m_{1}\left(I_{t}\right) m_{2}\left(A_{i}\right)\right]=a_{i} \cdot b+$ $\left[a_{i} \cdot(1-p \cdot b)+0 \cdot b\right]=a_{i} \cdot(1-p \cdot b+b)$,

for all $i \quad\{1, \quad 2, \quad \ldots, \quad p\}$.

(9)

It is interesting to finding out, according to the Conjunctive Rule, that the conflict of the above two sources does not depend on $m_{l}($.$) at all, but only on m_{2}($.$) , which is abnormal:$

$K_{12}=\sum_{i=1}^{p} \sum_{\substack{j=1 \\ j \neq i}}^{p} m_{1}\left(A_{i}\right) m_{2}\left(A_{j}\right)=\sum_{i=1}^{p} \sum_{\substack{j=1 \\ j \neq i}}^{p} a_{i} \cdot b=\sum_{i=1}^{p}(p-1) a_{i} \cdot b=(p-1) b \sum_{i=1}^{p} a_{i}=(p-1) b$.

Therefore even the feasibility of the Conjunctive Rule is questioned.

When we normalize, as in Dempster's Rule, by dividing all $m_{12}$ (.) masses by the common factor $1-K=1-p \cdot b+b$, we actually get: $\mathrm{m}_{1} \oplus \mathrm{m}_{2}=m_{1}$ ! So, $m_{2}($.$) makes no impact on$ the fusion result according to Dempster's Rule, which is not normal.

\section{MORE GENERAL CLASS OF COUNTER-EXAMPLES TO DEMPSTER'S RULE}

Let's consider $r+1$ sources: the previous $m_{l}($.$) and$ respectively various versions of the previous $m_{2}($.):

$\begin{array}{lccccc} & A_{1} & A_{2} & \ldots & A_{p} & I_{t} \\ m_{1} & a_{1} & a_{2} & \ldots & a_{p} & 0 \\ m_{21} & b_{1} & b_{1} & \ldots & b 1 & 1-p \cdot b_{1} \\ m_{22} & b_{2} & b_{2} & \ldots & b_{2} & 1-p \cdot b_{2}\end{array}$

$\begin{array}{llllll}m_{2 r} & b_{r} & b_{r} & \ldots & b_{r} & 1-p \cdot b_{r}\end{array}$

where of course all $a_{i} \in[0,1]$ and $a_{1}+a_{2}+\ldots+a_{p}=1$, also all $b_{j}$ and $1-p \cdot b_{j} \in[0,1]$, for $j \in\{1,2, \ldots, r\}$.

Now, if we combine $m_{1} \oplus m_{21} \oplus m_{22} \oplus \ldots \oplus m_{2 r}=m_{1}$. Therefore all $r$ sources $m_{21}(),. m_{22}(),. \ldots, m_{2 r}($.$) have no impact$ on the fusion result!

Interesting particular examples can be found in this case.

\section{SHORT GENERALIZATION OF DEZERT-TCHAMOVA COUNTER-EXAMPLE}

Let's consider four focal elements $A, B_{1}, B_{2}, B_{3}$, such that $A \cap B_{i}=\phi$ for $i \in\{1,2,3\}$, and $B_{1}, B_{2}, B_{3}$ are nested, i.e. $B_{1} \subset$ $B_{2} \subset B_{3}$, and two masses, where of course $b_{1}+b_{2}=1$ and $c_{1}+c_{2}+c_{3}=1$, and all $b_{1}, b_{2}, c_{1}, c_{2}, c_{3} \in[0,1]$ :

\begin{tabular}{ccccc} 
& $A$ & $B_{1}$ & $B_{2}$ & $B_{3}$ \\
$m_{1}$ & 0 & $b_{1}$ & $b_{2}$ & 0 \\
$m_{2}$ & $c_{1}$ & 0 & $c_{2}$ & $c_{3}$ \\
\hdashline$m_{12}$ & 0 & $b_{1}\left(1-c_{1}\right)$ & $b_{2}\left(1-c_{1}\right)$ & 0
\end{tabular}

and the conflict $K_{12}=c_{1}\left(b_{1}+b_{2}\right)=c_{1}$

$$
\begin{array}{lllll}
m_{D} & 0 & b_{1} & b_{2} & 0
\end{array}
$$

a) This generalization permits the usefulness of hybrid models, for example one may have the frame of discernment of exclusive elements $\{\mathrm{A}, \mathrm{B}, \mathrm{C}\}$, where $B_{1}=B \cap C, B_{2}=B$, and $B_{3}=B \cup C$.

b) Other interesting particular cases may be derived from this short generalization.

\section{PARTICULAR COUNTER-EXAMPLE TO THE CONJUNCTIVE} RULE AND DEMPSTER'S RULE 
For example let $\Theta=\{A, B, C\}$, in Shafer's model. We show that the conflicts between sources are not correctly reflected by the conjunctive rule, and that a certain nonvacuous non-uniform source is ignored by Dempster's rule.

Let's consider the masses:

$\begin{array}{llllll} & A & B & C & A \cup B \cup C \\ m_{1} & 1 & 0 & 0 & 0 & \text { (the most specific mass) } \\ m_{2} & 1 / 3 & 1 / 3 & 1 / 3 & 0 & \text { (very unspecific mass) } \\ m_{3} & 0.6 & 0.4 & 0 & 0 & \text { (mass between the very } \\ \text { unspecific and the most specific masses) } \\ m_{0} \quad 0.2 & 0.2 & 0.2 & 0.4 & \text { (not vacuous mass, not } \\ \text { uniform mass) }\end{array}$

Then the conflict $K_{10}=0.4$ between $m_{l}($.$) and m_{0}($.$) is the same$ as the conflict $K_{20}$ between $m_{2}($.$) and m_{0}($.$) , and similarly the$ same as the conflict $K_{30}$ between $m_{3}($.$) and m_{0}($.$) ,$

which is not normal, since $m_{l}($.$) is the most specific mass$ while $m_{2}($.$) is the most unspecific mass.$

Let's check other thing combining two sources using Dempster's rule:

$m_{1} \oplus m_{o}=m_{1}, m_{2} \oplus m_{o}=m_{2}, m_{3} \oplus m_{0}=m_{3}$,

which is not normal.

In order to get the "normal behavior" we combine $m_{l}($.$) and$ $m_{0}$ (.) with PCR5, and similarly for others: $m_{2}($.$) combined$ with $m_{0}($.$) , and m_{3}($.$) combined with m_{o}($.$) .$

In order to know what should have been the "normal behavior" for the conflict (the initial conflict was $K_{10}=0.4$ ), let's make a small change to $m_{0}($.$) as below:$

$\begin{array}{llllll} & A & B & C & A \cup B \cup C \\ m_{1} & 1 & 0 & 0 & 0 & \text { (the most specific mass) } \\ m_{2} & 1 / 3 & 1 / 3 & 1 / 3 & 0 & \text { (very unspecific mass) } \\ m_{3} & 0.6 & 0.4 & 0 & 0 & \text { (mass between the very } \\ \text { unspecific and the most specific masses) } \\ m_{0} & 0.3 & 0.2 & 0.1 & 0.4 & \text { (not vacuous mass, not } \\ \text { uniform mass) }\end{array}$

$K_{10}=0.30$

$K_{20}=0.40$

$K_{30}=0.34$

Now, the conflicts are different.

\section{CONCLUSION}

We showed in this paper that: first the conflict was the same, no matter what was one of the sources (and it is abnormal that a non-vacuous non-uniform source has no impact on the conflict), and second that the result using Dempster's rule is not all affected by a non-vacuous nonuniform source of information.

Normally, the most specific mass ( $b b a)$ should dominate the fusion result.

Therefore, the conflicts between sources are not correctly reflected by the conjunctive rule, and certain non-vacuous non-uniform sources are ignored by Dempster's rule in the fusion process.

\section{REFERENCES}

[1] F. Smarandache, J. Dezert, "Advances and Applications of DSmT for Information Fusion," Vols. I, II, III, American Res. Press, Rehoboth, 2004, 2006, respectively 2009; http://fs.gallup.unm.edu/DSmT.htm.

[2] J. Dezert, A. Tchamova, "On the behavior of Dempster's rule of combination," Presented at the spring school on Belief Functions Theory and Applications (BFTA), Autrans, France, 4-8 April 2011 (http://hal.archives-ouvertes.fr/hal-00577983/).

[3] J. Dezert., P. Wang, A. Tchamova, "On The Validity of DempsterShafer Theory." Proceedings of the International Conference of Information Fusion, Singapore, July, 2012.

[4] A. Tchamova., J. Dezert, "On the Behavior of Dempster's Rule of Combination and the Foundations of Dempster-Shafer Theory," (Best paper award), in Proc. of IEEE IS'2012, Sofia, Bulgaria, Sept. 6-8, 2012. 